\title{
Isotopic engineering in surface science and technology
}

\author{
A. A. Berezin \\ Department of Engineering Physics, McMaster University, Canada
}

\begin{abstract}
Isotopic Engineering (IE) is a generic term designating targeted use of specific isotopes to modify physical, chemical and technological properties of materials. Stable isotopes of the same chemical element differ in mass, diffusivity, magnetic properties and have different vibrational frequencies in crystalline matrices. This allows one to modify the bulk and surface properties of materials by using isotopic purification and/or changing relative abundances of stable isotopes. For example, natural carbon is a 99.1 percent mixture of $\mathrm{C}-12$ and $\mathrm{C}$ 13. Artificial diamonds grown from pure $\mathrm{C}-12$ (C-13 removed) show significant increase of thermal conductivity due to reduced phonon scattering in isotopically pure crystal lattice. Other examples of IE include light confinement at isotopic interfaces (isotopic fiberoptics), isotopic random number generators, etc. This paper provides an overview of possible applications of IE to modify surface and contact properties. We discuss isotopic effects in optical, electrical and mechanical properties, corrosion initiation and corrosion passivation, as well as a variety of applications of IE to micro and nanotechnology. Of the latter, we discuss isotopic information storage and the formation of isotopic quantum wells at the contact of isotopically different interfaces.

Keywords: isotopes, isotopic engineering, surfaces and interfaces, quantum effects, chaos, nanotechnology, tribology, quantum lubrication, corrosion, isotopic information technology.
\end{abstract}

\section{Introduction}

It is a common knowledge of today that physical, chemical and mechanical properties of material objects derive from the behavior of the constituting atoms 
and their interactions. Therefore, numerous key investigative techniques are designed to probe these properties at the atomic, nano and micro scales.

The same holds for surface and contact studies. For most areas of surface and interface science and technology, the physical effects at the nanoscale are of central relevance. Such powerful research tools as AFM (atomic force microscopy), neutron scattering, or secondary ion mass spectroscopy (SIMS), and a number of other surface methodologies are critically related to the surface structure at the level of individual atoms.

The majority of chemical elements exist in a form of two or more stable isotopes. Properties of different isotopes of the same chemical element are usually close, but not exactly the same. There are numerous isotopic effects known in physics and technology $[1,2]$. Consequently, it is a legitimate question to ask whether the whole gamut of bulk and surface properties and responses can be modified (in certain limits) through the purposeful isotopic ordering and isotopic purification. This idea gave birth to the specific area of (stable) isotopic engineering, IE [3-5]. The purpose of this paper is to discuss various aspects of IE and possible applications in the context of surface and contact phenomena. The prime emphasis is on quantum effects at micro and nano level.

Most of the effects discussed in this paper have not yet been experimentally tested and, hence, much of discussion remains at the level of hypotheses. The selection of the effects discussed is to a certain degree reflects personal preferences and interdisciplinary interests of the author, as well as his prior experience in the area of IE. Likewise, the bibliography is no way pretends to provide a comprehensive review. The latter aspect reflects the changing practice of the modern scientific writing which reflects the fact that modern Internet search engines, such as Google, allow to effectively compensate for the incompleteness of formal referencing.

\section{Stable isotopes in nature and technology}

In public perception the notion of isotopes is generally associated with radioactive isotopes and their applications. This association is quite understandable in view of such gigantic areas of economic activity as nuclear power generation or medical use of radioactive isotopes, as well as all sociopolitical tensions coming from nuclear weaponry proliferation. These perceptions to a certain degree overshadow in the public eyes the many roles of stable (non-radioactive) isotopes in science and technology. This renders the notion of "isotopicity" (science of stable isotopes) to a somewhat special position in material science and physical engineering. Some quantum effects mentioned in this paper are not necessarily centered on isotopic diversity, yet the use of isotopicity as a common thread allows for a certain unification of the context of this narrative. 


\subsection{Stable isotopes primer}

The fact that most (about 3/4) of all chemical elements have two or more stable isotopes is generally well known. Almost everyone has heard of a heavy water in which the light hydrogen is replaced by a doubly heavy deuterium. Properties of heavy and ordinary water are noticeably different (e.g., heavy water freezes at $+3.82{ }^{\circ} \mathrm{C}$ and boils at $101.4{ }^{\circ} \mathrm{C}$ ), but for heavier elements the difference in properties of isotopically different substances are not that large, and hence are often ignored. Yet, in some cases these, usually subtle, isotopic variations can be purposefully amplified (e.g., by some specific structuring) and gainfully explored for applications. This is true for the bulk properties of the materials, as well as for the effects related to the surface and interfaces between surfaces. Some examples, relevant to surface and contact phenomena are discussed below.

It may be a lucky play of nature that most technologically important elements have two or more stable isotopes, in particular, those elements which are central for micro and nano technology. For example, oxygen and silicon have 3 stable isotopes each, iron has 4 and zinc and germanium have 5. This opens the way for numerous avenues of IE through a purposeful isotopic structuring (for example, isotopic fiberoptics, which exploits isotopic variations in silicon dioxide [1-3]).

\subsubsection{Isotope separation and purification}

One of the central impediments to a broader exploitation of isotopic variations for engineering applications lies in the difficulty and high costs of isotope separation and isotopic purification. Chemically-based separation methods are based on differences in mass and diffusion coefficients between isotopes. Yet, they never achieve perfect purification of a particular isotope (there are always trace amounts or other isotopes) and costs of pure isotopes normally amount to hundreds dollars per gram. While these circumstances are discouraging for a wide front of experimental studies, they do not significantly affect theoretical and predictive studies of possible physical effects for isotopically structured materials. Theoretical physics is relatively cost-insensitive, as the whole history of science amply testifies.

\subsubsection{Physical basis for isotopic effects}

Numerous isotopic effects in physical, chemical and (less known) thermal and mechanical properties of materials generically come from several sources. Most (such as variations in diffusivity or lattice constants) come from the mass differences among isotopes, some other come from subtle differences in the position of atomic energy levels and differences in nuclear magnetic moments. The latter is the basis for the Nuclear Magnetic Resonance (NMR) methods which are broadly used in material studies and medical applications. 


\section{Isotopic engineering at macro, micro and nano scales}

Numerous aspects of IE were discussed earlier [1-5] and hence are only briefly mentioned here. Through the change of lattice constants and the presence of isotopic fluctuations, isotopic variations affect thermal, optical, as well as electrical properties of materials and their surfaces.

\subsection{Isotopic optical effects}

Isotopically engineered materials have slightly different refractive indices due to mass effect on the zero-point lattice vibrations [1]. This affects the surface reflectivity and changes the critical angle of the total internal reflection. One applicational consequence of that is the possibility of the isotopic light confinement and isotopic fiber-optics which is becoming one of the areas of IE.

\subsection{Thermal and electrical conductivity}

Thermal, and to some extent mechanical, properties of the materials depend on the lattice vibrations. Therefore, in theoretical solid state physics the overview of these properties usually presented as a branch of phonon physics (phonons are lattice vibrations). Electrical properties, such as conductivity and mobility, are affected by isotopic fluctuations and hence can be modified by isotopic purification [6] or specific isotopic structuring to reduce electron scattering and increase mean free path of conductivity electrons.

\section{Quantum, chaos and isotopic aspects of surface and contact physics}

Many, perhaps most, physical effects important for technological applications occur at surfaces or at interfaces between surfaces (contact effects). Almost all semiconductor electronics deals with quasi-2D (2-dimensional) systems, such as integrated circuits on chips, insulating thin films, thin film metal gates, etc. In chemical and electrical engineering surface aspects such as catalysis, absorption, corrosion, etc., play an equally important role. Isotopic purification and IE open another dimension for many of these areas.

\subsection{Isotopic surface modification and probing techniques}

There are several powerful surface micro fabrication techniques suitable for IE of surfaces. They can create isotopic patterns of almost any shape and structure at the resolution scale of individual atoms.

\subsubsection{Atomic manipulation techniques}

There are several technologies which can control trajectories of individual atoms and place them in required positions. These methods presently are hardly suitable for mass production, yet can be efficiently used for investigative and exploratory studies. 
4.1.1.1 Ion implantation techniques use collimated atomic beams and allow for a targeted implantation of particular isotopes at the desired locations [7]. Close technology to this is MBE (molecular beam epitaxy) which is excellently suited for atomic-scale structuring of specific isotopes.

4.1.1.2 Atomic force microscopy (AFM) is based on quantum tunneling effect [8]. It allows one to probe isotopic individuality of atoms at the surface and reveal the pattern (and its degree of randomness and/or ordering) which isotopes can form at the surface.

4.1.1.3 Secondary ion mass spectroscopy (SIMS) deals with atoms ejected from the surface upon ionic bombardment. The ejected atoms can be isotopically identified by the mass spectroscopy and, hence, the degree of isotopic purification of the surface can be established.

4.1.1.4 Non-thermal plasma deposition methods, such as CVD (chemical vapor deposition) allow for a relatively inexpensive way to produce isotopically engineered surfaces [9]. This may be especially useful for the fabrication of lager surfaces for various mechanical and electrical applications.

\subsection{Optical and magnetic isotopic surface effects}

Isotopic replacements can modify optical and magnetic properties of materials due to several mechanisms acting at atomic and nuclear level.

\subsubsection{Isotopic modification of optical indices}

At the physical basis the indices of refraction and reflection depend on the lattice constant. For example, uniform stress which changes the lattice constant is known to induce changes in the optical indices. Likewise, isotopic replacements produce variation of lattice constants due to the mass effect for the zero-point lattice vibrations. This, in turn, affects the optical indices [3] and band gaps [10]. 4.2.1.1 Phonon effects are especially sensitive to the isotopic replacements. The frequency of lattice vibrations is proportional to $1 / \mathrm{SQR}(\mathrm{M})$, and hence isotopic difference in the atomic mass leads to noticeable phonon frequency shift. For example, for C12 to C13 replacement, it amount to 0.04 (4 per cent), which is a difference with many macroscopically observable consequences (physics successfully deals with differences in parameters which are many orders of magnitude smaller, in fact, many important physical effects like spontaneous symmetry breaking are caused by energetically very weak seed effects).

4.2.1.2 Ballistic phonons are characterized by low lattice scattering. For isotopically purified surfaces and interfaces 2-dimensional phonon propagation can be of special interest in tribology studies. Sliding contact between surfaces normally generates phonons in a form of elastic waves which may have long mean free paths. This is due to diminished phonon scattering in low-dimensional isotopically pure conditions. Isotopic purification reduces scattering on isotopic fluctuations which is typical for isotopically mixed structures.

\subsubsection{Magnetic isotopic effects at interfaces}

In atoms magnetic effects have a two-fold origin, from electronic magnetic moments (spins) and nuclear magnetic moments. While electronic structure of 
atoms of different isotopes is the same, there are still slight differences in electronic energy levels due to different mass of nuclei. Nuclear magnetic moments for different isotopes are different due to different number of neutrons at different isotopes (each neutron has magnetic moment due to its spin). Interfaces between surfaces are quasi 2-dimensional systems and take smaller volume of the phase space than bulk (3-dimesional) systems. As a result, magnetic interactions at interfaces may become more long ranged than in bulk and this may result in the efficient amplification of magnetic effects at the contacts between surfaces.

4.2.2.1 Electronic magnetism at isotopically engineered interfaces can be "fine structured" to produce low frequency resonance effects and enhance energy transfer rates. These effects are observed in spin glasses, the latter presently fall into the research area called spintronics.

4.2.2.2 Nuclear magnetism being isotopically selective can play especially prominent role at contacts of two surfaces (quasi 2-dimensional systems) through the formation of network of magnetic moment interactions. The latter resembles artificial (spin glasses) neural networks [1] and can enhance energy exchange processes. This, in turn, may affect (reduce) friction between sliding surfaces acting in analogy with repulsive Van der Waals forces [8], leading to quasilubrication effect, as discussed below.

\subsection{Isotopic effects in surface corrosion}

The specific role of isotopic fluctuations in corrosion initiation was discussed earlier [11]. Formation of pitting corrosion phenomenologically appears as a random pattern with centers of its incipient formation been due to some form of defects or density fluctuations. For surface corrosion isotopic fluctuations (which lead to the spread of frequencies of local lattice vibrations, local phonons) may play a significant role as fluctuations in (quasi) 2-dimensional systems (surfaces and contacts) have generally higher relative statistical weight than in 3dimensional bulk situations.

\subsubsection{Isotopic purification in corrosion passivation}

Consequently, it is possible to suggest that in some specific cases, such as in microelectronic and micromechanical systems, isotopically purified surfaces and contacts may provide added advantage in terms of corrosion resistance. While for large scale structural and mechanical contacts the idea of isotopic corrosion passivation is unlikely technically and economically feasible, it may find some place in the areas of microelectronic, microelectromechanical (MEMS) and nanoelectromechanical (NEMS) devices, as well as in micro-medical devices such as body implants.

\subsection{Quantum and isotopic effects at surfaces and contacts}

Although friction at contacts usually discussed in the realm of a purely classical physics and mechanics, at the microscopic level quantum behavior can become significant and in some situations can become dominant. 


\subsubsection{Quantum lubrication}

As was already mentioned above, the repulsive forces at atomic scale may reduce friction coefficients and serve as form of "quantum lubrication". The origin of these forces lie in quantum electrodynamical fluctuations [8, 12-14] and for their studies the full apparatus of theoretical physics is to be employed.

4.4.1.1 Isotopic tribology can then be seen as a subset of the quantum lubrication effect. In this regard IE can possibly amplify quantum repulsive mechanisms. No experimental verification of this alleged isotopic effect is known to this author at this point. Of a special interest will be to relate these effects with earlier studies by Neumann and Wigner of quantum bound states in repulsive potentials [15]. In this regard, IE, as a tool for "fine quantum adjustments" (due to the general weakness of isotope effects) can serve as way to form such refined situations when quantum states contributing to quantum lubrication are becoming metastable (quasi-stationary).

4.4.1.2 Isotopic symmetry is an additional factor which can be introduced through a proper IE (e.g., symmetrical patterns of isotopes at the surface), such specific quantum effects as accidental degeneration (Zufallige Entartung) [16], may turn out to be both theoretically interesting and practically useful. In quantum physics degeneration traditionally signifies the situation when there are several distinguished quantum states have the same energy. Additional symmetry (in this case due to symmetrical isotopic patterning) raises the level of degeneration and makes it "accidental" $[16,17]$. In the opinion of the present author, quantum effects in surface tribology (including "quantum lubrication") are largely unexplored and potentially constitute a promising area of research and development.

4.4.1.3 Isotopic quantum wells which can be purposefully created by IE [18] or through the spontaneous isotopic fractionation due to phonon interactions [19] can turn up to be an alternative basis of quantum lubrication effect. More details on that are below (section 4.4.5.1).

\subsubsection{Isotopic interfaces and electrostatics}

Electrostatic effects play a significant role at the interfaces and contacts between surfaces. Numerous forms of frictional charging are often discussed in quantum mechanical context [20]. Isotopicity can affect these processes in several ways, such as formation of electronic trapping centers on isotopic fluctuations, change of electronic mobility [6], or affecting characteristic times of electronic delocalization.

\subsubsection{Thermal effects and isotopic engineering}

Isotopic structuring and isotopic purification may significantly affect thermal conductivity [1], up to a factor 2 to 3 as was observed for isotopically pure artificial diamonds. As a consequence of that, heat sinking and frictional motion between surfaces can be affected. Changes in thermal conductivity can, in turn, modify thermal expansion due to changes in local heating at microscopic level. These effects may have some bearing for MEMS, NEMS and medical biotechnology. 
4.4.3.1 Hopping conductivity is an alternative form of electrical conductivity (to distinguish it from drift and diffusion conductivity) which is typical for materials with microscopic trapping centers. It proceeds by the mechanism of quantum tunneling between trapping sites. Hence, isotopic clustering and isotopic fluctuations acting as trapping sites can affect the dynamics of hopping conductivity.

4.4.3.2 Poole-Frenkel effect is thermal ionization enhanced by electric fields in the material due to the reduction of the potential barrier for electron release from the well (in this case - isotopic quantum well). Electrons trapped at isotopic fluctuations can produce random electric fields affecting the effective heights of the potential barriers. Each individual trapped electron in a potential well is affected by the fields of other electrons. Hence, the whole system here falls into the class of cooperative phenomena (all particles affect each other). In tribology context, the collective delocalization of trapped electrons can enhance quantum lubrication effect.

\subsubsection{Isotopic information technology}

Practically all electronic information technology existing today uses surfaces of materials, as well as contacts, for the information processing and storage. Integrated circuits (IC) have thin film grids deposited on semiconductor chips. Flush memories have arrays of thin field effect transistors (FET) forming quasi2-dimensional networks on chips. Ability of classically distinguishable isotopes to be used as information bits [1] can vastly increase the packing information density. This primarily concerns the ROM-type memory (permanent, Read Only Memories) type of information storage, but also can be upgraded to a dynamic (changeable) memory storage using magnetic moments of specific isotopes. The latter can be re-switched between up and down spin states which can be used to store binary bits and change them by outside pulses. Likewise, the developing area of quantum computing [21] also uses systems with isotopes with non-zero nuclear magnetic moments which in magnetic fields can exhibit bistability and polystability effects.

\subsubsection{Repulsive quantum effects and contact friction}

In certain situation there may be repulsive forces forming between surfaces at contacts. In case of sliding contacts these effects may reduce the effective friction coefficients thus serving as a form of inter-surface lubrication. The origins of these repulsive forces lie in quantum mechanical and quantum electrodynamical effects [14].

4.4.5.1 Isotopic quantum wells and quantum lubrication can be explained within the framework of standard non-relativistic quantum mechanics. Impurity or isotopic structuring at contact areas between surfaces may lead to the formation of potential wells in the form of quantum dots or isotopic quantum wells [18]. An electron trapped in such a well creates a pressure on the walls of the well. This pressure, in turn, generates repulsive forces at the interfaces thus, in effects, reducing friction coefficient between the sliding surfaces. The origin of this mechanism is purely quantum. It is a direct result of the Uncertainty Principle of Quantum Physics (Heisenberg principle) which shows that the 
energy levels of a trapped particle scale as the inverse square of the size of the quantum well.

4.4.5.2 Repulsive van der Waals effect, also known as repulsive CasimirLifschitz effect $[8,12-14]$ is not exclusively related to isotopic diversity. It is based on quantum electrodynamics and its explanation involves pressure effects from zero-point vibrations of virtual electromagnetic fields in the contact region. The play goes on the differences of the dielectric constants of both surfaces and the thin layer of the liquid medium between them. Experimental and theoretical studies of quantum lubrication effects are now in progress in a few laboratories over the world.

\subsubsection{Energy transfer effects}

Another class of largely unexplored possibilities may be related to energy transfer and electron hopping effects between nano-scale isotopic clusters at the interface region $[18,19]$. Resonance energy transfer between clusters (or isotopic fluctuations) can affect the inter-surface tension and change (reduce or increase) the effects of quantum lubrication. Possible areas of application are, again, most likely related to small-scale devices like MEMS an bio- medical electronics.

\subsection{Effects of chaos and self-organization in surface isotopic engineering}

Recent developments in material physics ("soft state physics") make a significant emphasis on the processes of chaotic dynamics and spontaneous selforganization. Isotopic diversity provides an additional dimension to that.

\subsubsection{Isotopic chaos and isotopic self-organization}

From the standpoint of quantum physics isotopes of the same chemical elements are macroscopically (classically) distinguishable objects. This differ them from elementary particles (electrons, protons, etc) and identical atoms (same isotopes) which are, according to principles of quantum physics, are indistinguishable (e.g., when two electrons collide and then fly apart, we cannot say "which is which"). This aspect (isotopic distinguishability) results in some differences in chaotic phenomena related to isotopic diversity [22]. This has a special impact for the processes of self-organization and pattern formation.

\subsubsection{Chaos and chaotic dynamics}

While the term "chaos" comes from antiquity and has a general meaning of disorder, in modern physics it has a more focused delineation within the realm of non-linear dynamics. Two prime concepts in the theory of chaos are strange attractors [22] and sensitivity to initial conditions (the "Butterfly effect"). Another key aspect in chaos theory is a dichotomy of order and disorder, chaotic dynamical systems are prone with multiplicity of order-disorder transitions. Within this set, systems with isotopic diversity have much more statistical degrees of freedom than isotopically uniform systems of the same size, and hence show a greater variety of scenarios for order-disorder transitions. At the same time, many effects at nano, micro, meso and macro scales (such as pattern formation and positional correlations) have both chaotic and quantum aspects. 
4.5.2.1 Order-disorder transitions in material sciences are often more easily observed at the surface and contacts than in the bulk. Such surface probing techniques as AFM can reveal isotopic patterns at the surface. Of a specific interest here is the entropy assessment of isotopic patterns at the surface. Its formation and evolution can be modeled on the basis of maximum entropy principle of Jaynes [23], which is bayed on iterative (Bayesian) statistics with links to the theory of information.

4.5.2.2 Isotopic randomness is inherently contradictory issue [2, 22]. To what extent can the distribution of isotopes be considered random or having some long and/or short distant correlations? For example, can AFM of isotopes at the surface detect such isotopic positional correlations? These questions appeal to the very definition of randomness which may be a mathematically open issue (for example, nobody yet proved that the infinite string of digits of pi = $3.14159 .$. is truly "random" and there are no "hidden correlations" in it). To a degree, these questions can be deepened by comparing random (quasi-random?) strings produced by isotopic random generators [2] with strings from other types of random number generators.

4.5.2.3 Gibbs paradox gives another angle to these issues. In thermodynamically non-equilibrium system (of which sliding surfaces is just one example) the dynamics of pattern formation at atomic level is driven by (local) energy minimization as well as entropic constrains. Distinguishability of isotopes within classical statistical physics leads to another controversy related to Gibbs paradox (nonadditivity of entropy at mixing of likewise gases). According to Jaynes [23], a great deal of confusion arises over the issue as to whether entropy of mixing of isotopes should or should not be included in entropy assessment. This aspect generates further discussion with an attempt to resolve it within quantum physics and quantum thermodynamics [24].

4.5.2.4 Gravity quantum effects refer to spontaneous reduction of the wave functions and can play role at meso and micro scales typical for surfaces and contacts in MEMS-type devices. Penrose [25] and other authors have discussed effects of gravity (general relativity) at micro scale objects. Isotopic sensitivity of these effects comes from isotopic mass difference which affects the rate of spontaneous quantum reductions. This may shift the balance between localized and delocalized states in contact areas which, in turn, may affect the dynamics of quantum lubrication effects.

4.5.2.5 Quantum entanglement effects have recently went into a focus in variety of areas from quantum informatics to nano-technology and biomedical sciences. For surfaces and contacts of MEMS-scale devices it is another exploratory area for micro- and nano-tribology. In this regard isotopic purification and IE can be instrumental for strengthening of quantum coherency and connectivity of the interfacial system.

\section{Conclusion}

While the application of quantum physics to surface and contact mechanical effects is it its incipient stages, some advanced models of quantum lubrications 
have been recently discussed [26]. The examples of quantum and chaotic aspects of contact physics mentioned in the present article bear heuristic character as possible new directions of research. Not necessarily all these examples will be actively pursued in further developments and, conversely, some new, yet unexpected, aspects may come forward. Likewise, not all physical effects reviewed above are singularly based on isotopic diversity. But in spite of occasional (yet, deliberate) over-extrapolation of the presumed role of isotopic effects, an appeal to isotopicity as a common theme provides a conceptual synchronicity for the discussion. As with any hypothetical discussion paper, the author assumes no responsibility for the completeness of the list of the proposed effects. The main trust of this position paper was to delineate isotopic surface engineering as a new area of surface science. While isotopicity takes somewhat specific niche in physics and material science, it also can serve as a research tool and versatile probe for a variety of physical effects.

\section{References}

[1] Berezin, A.A., "Isotopicity: Implications and Applications", Interdisciplinary Science Reviews, 17 (1), pp. 74-80, 1992.

[2] Berezin, A.A. "Isotopic diversity in natural and engineering design", In "Design and Nature II", editors: Collins, M., Brebbia, C.A. WIT Press, Southampton, Boston, pp. 411-419, 2004.

[3] Berezin, A.A., "Isotopic Engineering (Perspectives)", Journal of the Physics and Chemistry of Solids, 50, pp. 5-8, 1989.

[4] Plekhanov, V.G., "Fundamentals and applications of isotope effects in solids", Progress in Material Science, 51, pp. 287-426, 2006.

[5] F. Simon, F., Kramberger, Ch., Pfeiffer, R., Kuzmany, H., V. Zolyomi, V., Kurti, J., Singer. P.M. \& Alloul, H., "Isotope Engineering of Carbon Nanotube Systems", Physical Review Letters, 95(1), 017401, 2005.

[6] Berezin, A.A., "Isotopic Disorders as a Limiting Factor for the Mobility of Charge Carriers", Chemical Physics Letters, 110, pp. 385-387, 1984.

[7] Ibrahim, A.M. \& Berezin, A.A., "Synthesis of Buried Insulating Layers in Silicon by Ion Implantation" (Review), Materials Chemistry and Physics, 31, pp. 285-300, 1992.

[8] Feiler, A.A., Bergstrom, L. \& and Rutland, M.W., "Superlubricity Using Repulsive van der Waals Forces”, Langmuir, 24, pp. 2274-2276, 2008.

[9] Chen, X., Chang, J.S., Berezin, A.A., Ono, S. \& Teii, S., "Isotope Effects in Amorphous Silicon Thin Films Produced by Ar-SiH4-D2 Plasma Chemical Vapor Deposition Method", Journal of Applied Physics, 69, pp. 1678-1686, 1991.

[10] Berezin A.A. \& Ibrahim, A.M., "Semiempirical Model of Isotopic Shifts of the Band Gap", Physical Review B, 43, pp. 9259-9261, 1991.

[11] Berezin, A.A., "Isotopic Effects and Corrosion of Materials", Materials Physics and Chemistry, 34, pp. 91-100, 1993.

[12] Munday, J.N., Capasso, F. \& Parsegian, V.A., "Measured long-range repulsive Casimir-Lifshitz forces", Nature, 457, pp. 170-173, 2009. 
[13] Cranston, E.D. \& Gray, D.G., "Formation of cellulose-based electrostatic layer-by-layer films in a magnetic field", Science and Technology of Advanced Materials, 7, pp. 319-321, 2006.

[14] Lamoreaux, S.K., "Quantum force turns repulsive”, Nature, 457, pp. 156157, 2009.

[15] von Neumann, J. \& Wigner E.P., "Uber merkwurdige diskrete Eigenwerte", Physikalische Zeitschrift, 30, pp. 465-467, 1929.

[16] Balantekin, A.B., "Accidental degeneration and supersymmetric quantum mechanics", Annals of Physics, 164, pp. 277-287, 1985.

[17] Berezin, A.A., "Isotopic Lattice Symmetry: An Unexplored Area of Research", Structural Chemistry, 3, pp. 169-174, 1992.

[18] Berezin, A.A., "Localized Electron Levels in Isotopically Ordered Crystals (Isotopic Quantum Wells)", Physica Status Solidi (b), 144, pp. 727-732, 1987.

[19] Goldman, C. \& Berezin, A.A., "Isotopic Fractionation by Phonon Induced Interactions", Physical Review B, 51, pp. 12361-12368, 1995.

[20] Berezin, A.A., "Electrification of Solid Materials", In: Handbook of Electrostatics, Chang, J.S., Kelly, A.J. \& Crowley, J.M. (eds), New York: Marcel Dekker, pp. 25-38, 1995.

[21] Berezin, A.A., "Quantum computing and security of information systems", in Safety and Security Engineering II, Guarascio, M., Brebbia, C.A. \& Garzia, F. (eds), (Safe II: Second International Conference on Safety and Security Engineering), pp. 149-159, 2007.

[22] Berezin, A.A., "Isotopic Relative of Strange Attractors", Physics Letters A, 161, pp. 295-300, 1991.

[23] Jaynes, E. T., "The Gibbs Paradox", in Maximum-Entropy and Bayesian Methods, Smith, C.R., Erickson, G.J. \& Neudorfer, P.O. (eds.), Kluwer Academic Publishers, Dordrecht, Holland, pp. 1-22, 1992.

[24] Allahverdyan, A.E. \& Nieuwenhuizen, T.M., "Explanation of the Gibbs paradox within the framework of quantum thermodynamics", Physical Review E, 73, Article No. 066119 (15 pages), 2006.

[25] Penrose, R., “On Gravity's Role in Quantum State Reduction”, General Relativity and Gravitation, 28(5), pp. 581-600, 1996.

[26] Feldmann, T. \& Kosloff, R., "Quantum lubrication: Suppression of friction in a first-principles four-stroke heat engine", Physical Review E, 73, Article 025107(R), (4 pages), 2006. 